\title{
Construction of Early Warning System for Urban Subway Police Equipment Based on Mobile Phone
}

\author{
Liang $\mathrm{Hu}^{*}$, Hongmei Yu, Jin Wen and Jiangqing Liu \\ Department of humanities and management, Jiangxi Police College, Nanchang, China \\ ${ }^{*}$ Corresponding author
}

\begin{abstract}
With the rapid development of the construction of large cities in China, the population density has soared, and the subway is an important transportation tool in modern cities. In order to solve the problem of fault information transmission, the paper adopts the technology of seamless connection between the smart phone and WiFi network, and constructs a remote fault alarm platform for Metro equipment. Experimental results show that the platform has the characteristics of real-time, high stability, low cost and easy maintenance, information can be sent at the same time to different types of terminals, to ensure efficient and reliable data transmission based on the dynamic monitoring of the subway equipment, and provide a reliable guarantee for subway safety, time and efficiency.
\end{abstract}

Keywords-urban subway; public security; equipment management; mobile phone

\section{INTRODUCTION}

The subway transportation is the public transportation infrastructure in the city, it is the important item of the modern city construction, and it is also the booming industry. Subway traffic once the project is put into operation, the normal operation of the whole system must maintain uninterrupted, and the whole system's normal operation, must be based on the premise and guarantee for the safe operation of equipment, failure of any equipment, are likely to be a major safety problem[1][2]. Before the use of information technology, the company equipment management and equipment maintenance management work is basically using the traditional manual management, low efficiency of management, the labor cost is relatively high, the equipment failure does not respond in a timely manner, affecting the safety of Metro operation. Therefore, to realize the fault information by means of timely alarm, establish efficient and quick fault alarm platform, solve the equipment failure problems in the shortest possible time, to avoid greater losses, become an important research direction in the field of subway[3][4][5].

$3 G$ technology is also known as the third generation mobile communication, its $20 \mathrm{MHz}$ spectrum bandwidth can reach $100 \mathrm{Mbit} / \mathrm{s}$ 50Mbit/s transmission rate, and in the case of $350 \mathrm{~km} / \mathrm{s}$ to mobile users to provide $100 \mathrm{kbit} / \mathrm{S}$ rate access services. The technology supports unpaired or paired spectrum, flexible configuration of $1.25 \mathrm{MHz}$ to $20 \mathrm{MHz}$ between different broadband. WiFi is a personal computer, handheld devices (such as pad, mobile phone and other terminal) connected to wireless technology, is a brand of wireless network communication technology, held by the Wi-Fi alliance, WiFi is an important part of WLAN. With the development of WiFi technology and the continuous improvement of standards, WiFi technology is widely used in various fields, the application of foreign WiFi in the subway is relatively more, but the country has just started. 14:20 on November 28, 2014, Chinese first opened WiFi service passenger train from Guangzhou to Hong Kong T809 times Kowloon through train from Guangzhou East Railway Station, mark China railway began the era of WiFi. Therefore, this paper designed the scheme of $3 \mathrm{G}$ and $\mathrm{WiFi}$ alarm device based on the subway, the subway can be achieved on the equipment fault information is transmitted to the monitoring center station, do not need to set up special communication network, artificial fault reporting into automatically send alarm information, not only can save manpower and time, but also improve the efficiency of equipment alarm platform[6][7].

\section{SYSTEM ARCHITECTURE DESIGN}

In the subway system, the alarm system is the most obvious limitation of objective conditions. Therefore, the application of wireless communication technology plays a key role. Metro train monitoring system for wireless technology has the following requirements: high data transfer rate, support for the state of motion, the wireless network to strip coverage, the need for roaming switch, reliability. The subway equipment fault alarm platform system based on mobile phone mainly includes subway equipment, mobile phone terminal and subway server. Through the mobile phone collection of subway equipment fault information, the use of $\mathrm{WiFi}$ mobile phone access to WLAN, the information will be sent to the subway server, and then by the subway server through the 3G network transmission to the subway monitoring center[8].

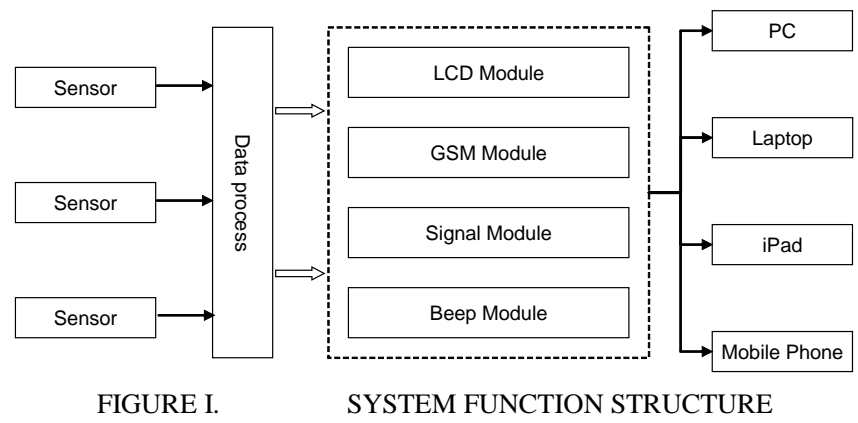

The system uses GSM wireless module of the SMS function, to monitor various scene that need real-time fault alarm in time to responsible personnel information. The function structure of the system is shown in Figure 1. The system is mainly responsible for receiving the fault information, 
extract telephone and alarm information corresponding to the mobile phone terminal through the GSM module that is sent to the responsible person in a timely manner, so that the responsible person can grasp the responsibility in the fault information and process the equipment failure.

In this paper, based on the $\mathrm{B} / \mathrm{S}$ architecture, make full use of the user interface is friendly, high efficiency and mobility, the intelligent mobile phone as the mobile client, mobile phone play data processing ability, through WiFi and $3 \mathrm{G}$ network, the subway equipment fault information is transmitted to the monitoring center to complete the subway, subway equipment failure information input, query, statistics analysis and alarm functions.

\section{SYSTEM NETWORK ARCHITECTURE}

In the subway monitoring system, the train monitoring system is restricted by objective conditions. Therefore, the application of wireless communication technology plays a key role. The network of the system adopts the way of combining wired optical network and AN. The subway operation control center is connected with the AP of the subway sub stations, subway sub stations and tunnels, and the AP in the fixed tunnel and the AP on the high-speed running subway are connected by WLAN mode, as shown in Figure 2.

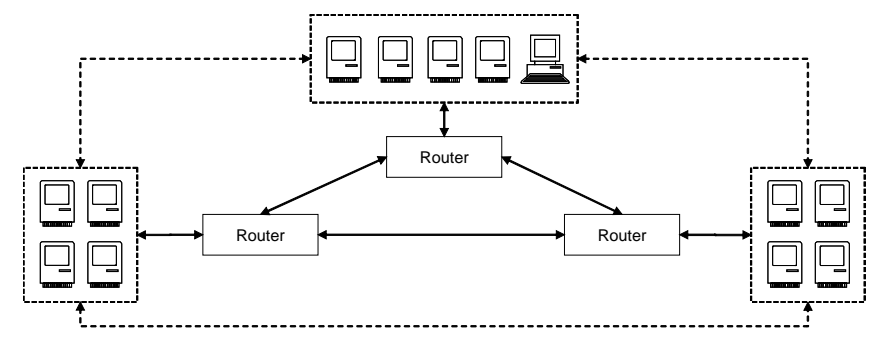

FIGURE II.

SYSTEM NETWORK ARCHITECTURE

In this WLAN environment, there are a number of data transmission and acceptance of the device, that is wireless access point AP. In essence, the access point acts as a LAN hub. Typically, a AP is valid in the range of 250 meters, so in the subway tunnel separated by about 2 meters to install a AP. As the microwave is a straight line, in a large angle, the distance may be less than 20 meters apart, should be based on the actual situation. The access point AP to receive the train through the signal of the wireless network transmission, and then transmit to the server, the server through data fusion, to remove redundant data, then the data into a steady stream of data, the server will send the data flow to the control center through the switch.

The directional antenna is used in the subway tunnel, and the tunnel has a strong reflection effect on the wireless. The same frequency interference refers to the use of the same frequency band of 2 AP in the tunnel, the distant AP wireless signal to the nearby A P noise. The solution is to try not to use the same frequency band adjacent to the AP; set the appropriate AP signal strength, not too strong; set the AP noise threshold, filter out the weak wireless signal. Multi path reflection is the same wireless signal source after a number of reflection in the tunnel at a certain point, the wireless signal phase is just the opposite, after superposition is close to zero. The method is to adopt double receiving antenna.

\section{Mobile Client Data Processing TeChNOLOgY}

Because the CPU data processing speed of mobile phone is relatively slow, access to the data of long reaction time, to improve data access speed and display system, the data saved as XML format, stored in the NXD, and each data as a node, forming a data oriented XML document processing. Each data is stored in NXD in the form of a single node, which can realize the rapid access of data, and can be used to update the individual nodes.

Intelligent mobile phone data exchange server via WLAN and using WiFi technology in the network, weak signal, low data rate, using asynchronous parallel transmission technology, the user submitted after the input interface returns directly by the system, the main thread in the background of the data package, and assigned to a data transmission thread that specifically by WLAN transmission to the server, mobile phone successfully received signal server returns after transmission thread releases the invalid data, re-enters a wait state.

\section{Database Processing Technology of Metro SERVER}

The server receives and processes the data, data exchange and intelligent mobile phone client, in order to improve the response speed, the server uses the thread pool technology of asynchronous parallel processing data received, and the time to release the disk occupying thread. With the phone connected to the thread, set the independent time wheel, if not received within a few seconds of data, it is determined that the connection is idle, you need to disconnect and release the thread.

As the mobile phone system memory is small, CPU processing speed is relatively slow, and the subway server memory, processing speed, data processing and analysis of the main functions in the server. Therefore, the technology of subway server can effectively play its performance and data processing ability.

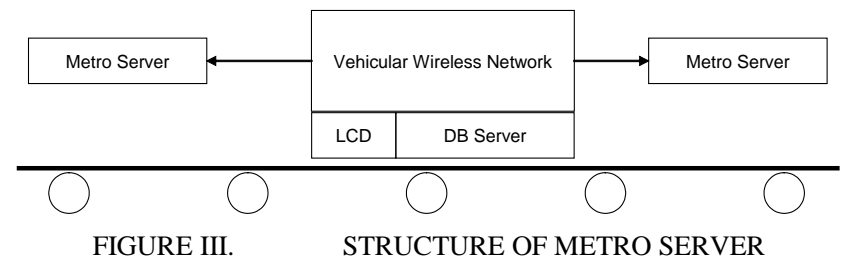

\section{DISCUSSION AND FUTURE WORK}

China's subway transportation is in the stage of rapid development, the scale of subway traffic will be expanded rapidly, and the flow of people will be more and more complex. Therefore, the use of intelligent mobile phone alarm technology platform of subway equipment based on wireless equipment can realize the management of the subway, and ensure the equipment fault information real-time transmission 
to the subway station on the subway management, improve the safe and stable operation of great significance.

\section{ACKNOWLEDGMENT}

This author's work is supported by JiangXi Research on teaching reform of higher education(JXJG-15-19-3), JiangXi Science and technology research project of Education Department(GJJ151193), JiangXi Social Science Planning Projects during the 12th Five-Year Plan(14TQ05), JiangXi University Party Building Project(16DJQN065).

\section{REFERENCES}

[1] Yang Xingyu. GPRS wireless transmission application in remote image monitoring system[J]. Microcomputer Information, 2005,21 (3): 64-65.

[2] Jiang Yi, Yang Chongjun, Liu Donglin et al. Design and implementation of public transport system based on PDA[J]. Computer Application Research, 2007, (1): 280-282.

[3] Liu Bohong. Discussion on metro equipment maintenance management [J]. Modern Urban Rail Transit, 2009,8:32-33.

[4] Xiong Ziyao. Design and implementation of PDA Based Railway fault prediction system[J]. Computer Knowledge and Technology, 2009,6:2427.

[5] Ning Chen. Research on AP handover mechanism based on throughput in WLAN[J]. Science Technology and Engineering, 2006 (2): 201-205.

[6] Zhang Hui. Analysis of wireless local area network technology and its application in rail transit construction[J]. Railway Communication Signal Engineering Technology, 2009, 6(02): 44-60.

[7] Liu Shufang. Application of root cause analysis of equipment failure in Metro[J]. Urban Rapid Rail Transit, 2010,2:21-23.

[8] Wang Di. Design and implementation of Nanjing Metro Enterprise Asset Management[J]. Urban Rapid Rail Transit, 2011, 10: 1-14. 